\title{
Storm-time electron flux precipitation in the inner radiation belt caused by wave-particle interactions
}

\author{
H. Tadokoro ${ }^{1}$, F. Tsuchiya ${ }^{1}$, Y. Miyoshi ${ }^{2}$, Y. Katoh ${ }^{1}$, A. Morioka ${ }^{1}$, and H. Misawa ${ }^{1}$ \\ ${ }^{1}$ Planetary Plasma and Atmospheric Research Center, Tohoku University, Aoba-ku, Sendai, 980-8578, Japan \\ ${ }^{2}$ Solar-Terrestrial Environment Laboratory, Nagoya University, Nagoya, 464-8601, Japan
}

Received: 29 July 2008 - Revised: 27 February 2009 - Accepted: 24 March 2009 - Published: 7 April 2009

\begin{abstract}
It has been believed that electrons in the inner belt do not show the dynamical variation during magnetic storms except for great magnetic storms. However, Tadokoro et al. (2007) recently disclosed that low-altitude electrons in the inner belt frequently show flux variations during storms (Storm Time inner belt Electron Enhancement at the Low altitude (STEEL)). This paper investigates a possible mechanism explaining STEEL during small and moderate storms, and shows that it is caused not by radial transport processes but by pitch angle scattering through wave-particle interactions. The waves related to wave-particle interactions are attributed to be banded whistler mode waves around $30 \mathrm{kHz}$ observed in the inner magnetosphere by the Akebono satellite. The estimated pitch angle distribution based on a numerical calculation is roughly consistent with the observed results.
\end{abstract}

Keywords. Magnetospheric physics (Energetic particles, precipitating; Energetic particles, trapped; Storms and substorms)

\section{Introduction}

It is generally believed that energetic electrons in the inner radiation belt are relatively insensitive to magnetic storms, while energetic electrons in the outer radiation belt show huge dynamical variation during storms (e.g., Friedel et al., 2002; Miyoshi et al., 2004; Miyoshi and Kataoka, 2005; Shprits et al., 2008a, b). A source mechanism of inner belt electrons has been considered to be only the injection from the outer belt at the time of a great magnetic storm (e.g., Bostrom et al., 1970; Gusev et al., 1995; Baker et al.,

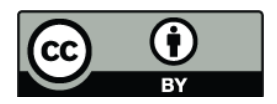

Correspondence to: H. Tadokoro (syougun@pparc.geophys.tohoku.ac.jp)
2004). The principal loss mechanism of electrons in the inner belt is pitch angle scattering due to Coulomb collisions (e.g., Walt and Newkirk, 1966) and wave-particle interactions (e.g., Lyons et al., 1972). In fact, the regular precipitation of energetic electrons $(\sim$ several tens of $\mathrm{keV})$ in the inner belt has been observed by low altitude satellites (e.g., Imhof et al., 1974; Inan et al., 1982). Recent articles reported that whistler-induced electron precipitation (WEP) from lightning is a significant inner radiation belt loss process at more than $100 \mathrm{keV}$ (e.g., Rodger et al., 2003, 2007). Abel and Thorne (1998a, b, 1999) estimated precipitation lifetimes due to wave-particle interactions with plasmaspheric hiss, lightning-generated whistler mode waves, and VLF transmitter sources as well as Coulomb collisions.

There are only a few reports about the responses of inner belt electrons during magnetic storms. Kikuchi and Evans (1989) showed sudden enhancement of low Earth orbit electrons $(>30 \mathrm{keV})$ at the lower $L(\sim 1.3)$ during a great magnetic storm. Morioka et al. (2001) and Miyoshi et al. (2002) reported that an enhancement of $300 \mathrm{keV}$ electron flux in the inner belt $(L \sim 2)$ at a NOAA altitude is synchronized with the onset of the outer belt electron decrease during the storm main phase. Datlowe (2006) showed that energy spectra of energetic electrons in the inner belt $(L=1.2)$ have two types: quiet time and storm time spectra corresponding to two different scattering mechanisms during the quiet time and magnetic storm time, respectively. Tadokoro et al. (2007) examined characteristics of Storm Time inner belt Electron Enhancement at the Low Earth orbit (STEEL), especially electrons at $L=2.0$ where is the outer edge of inner belt at $300 \mathrm{keV}$ electrons as discussed in the paper, during small and moderate magnetic storms $(-100 \mathrm{nT}<$ minimum $D_{s t}<-30 \mathrm{nT}$ ), and clarified that the electron flux of the inner belt at a low altitude increases by over one order of magnitude during the main phase of storms, and the duration of the enhancement is approximately 1 day. They focused on small and moderate storms which are not accompanied by

Published by Copernicus Publications on behalf of the European Geosciences Union. 


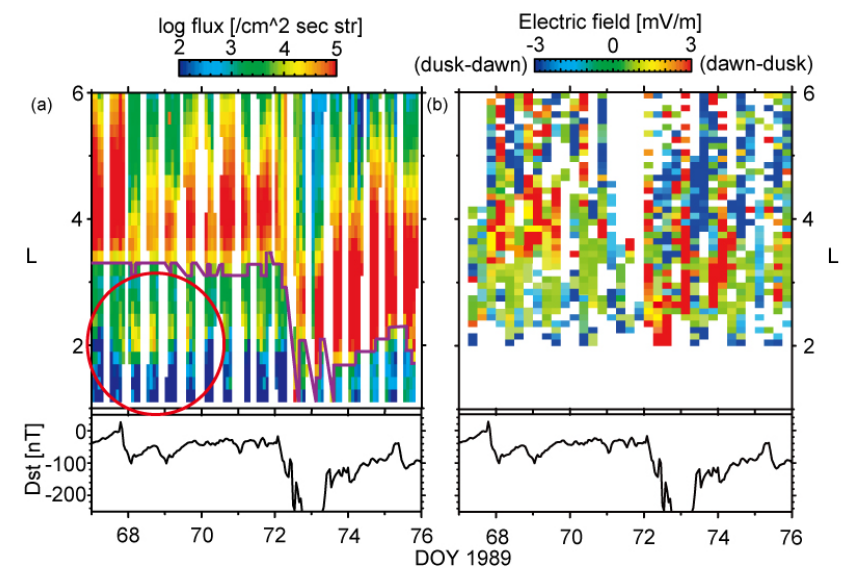

Fig. 1. L-t diagram of $300 \mathrm{keV}$ electron flux observed by NOAA (a) and variation of convection electric fields at the magnetic equator (b) during the period from day 67 to day 76 of 1989. The bottom panels show the $D_{s t}$ index.

injection from the outer belt, and suggested pitch angle scattering in the inner magnetosphere due to wave-particle interactions as a possible mechanism causing STEEL. An unsolved problem is to clarify how wave-particle interactions work to STEEL. In the present study, we investigate an electron scattering process in the inner belt due to wave-particle interactions, and also estimate flux of electron precipitation in order to identify a possible mechanism for STEEL.

\section{Observations}

The NOAA 12 satellite was in a sun-synchronous orbit with an inclination of $98^{\circ}$, an altitude of $815 \mathrm{~km}$, and an orbital period of $102 \mathrm{~min}$. The local times of its ascending and descending nodes were $19.5 \mathrm{~h}$ (dusk side) and $7.5 \mathrm{~h}$ (dawn side), respectively. The Medium Energy Proton Electron Detector (MEPED) onboard the NOAA 12 satellite measured electron counts in three energy channels (E1: 30-1100, E2: 100-1100, and E3: $300-1100 \mathrm{keV})$, and proton counts in five energy channels (P1: 30-80, P2: 80-250, P3: 250-800, P4: 800-2500, and P5: $>2500 \mathrm{keV}$ ). The MEPED is made up to two sensors; one, called the $0^{\circ}$ sensor, that views radially outward along the vector from the Earth to the satellite, and the other, called the $90^{\circ}$ sensor, views in a direction perpendicular to the $0^{\circ}$ sensor. The details of the MEPED are described in Raben et al. (1995). These sensors sometimes suffer contamination from different energetic particles. Tadokoro et al. (2007) examined the possibility of contamination at STEEL, and concluded that electron flux enhancements at STEEL are not due to contamination. Electron and ion distributions at the NOAA altitude depend on geographic longitude because of the magnetic anomaly caused by the offset of the terrestrial magnetic field. Therefore, observed electrons consist of electrons in the bounce loss cone, the drift loss cone, and the trapped region. Following a method of Blake et al. (2001), we restrict the data in the drift loss cone and in the trapped region to geographic longitude ranging from $180^{\circ}$ to $280^{\circ}$ in the Northern Hemisphere, and from $180^{\circ}$ to $250^{\circ}$ in the Southern Hemisphere, and from $300^{\circ}$ to $50^{\circ}$ in the Southern Hemisphere (South Atlantic Anomaly). The electrons in these selected regions are almost insensitive to the anomaly of geomagnetic fields and have approximately the same inner belt flux level.

The Akebono (EXOS-D) satellite was launched in 1989 into a quasi-polar orbit with an inclination of $75^{\circ}$, an orbital period of $212 \mathrm{~min}$, and an initial perigee and apogee of $274 \mathrm{~km}$ and $10500 \mathrm{~km}$. The Plasma Wave and Sounder Experiment (PWS) onboard the Akebono satellite detects electric fields in the frequency range from $20 \mathrm{kHz}$ to $5.12 \mathrm{MHz}$ (Oya et al., 1990). The Very Low Frequency Plasma Wave Detector (VLF) detects electric and magnetic fields in the frequency range from $3.16 \mathrm{~Hz}$ to $17.8 \mathrm{kHz}$ (Kimura et al., 1990). The Electric Field Detector (EFD) measures DCelectric fields using double probe method (Hayakawa et al., 1990).

\section{Results and discussion}

\subsection{Radial transport process}

Figure 1a shows the L-t diagram of the $2 \mathrm{hr}$-averaged electron flux at $300-1100 \mathrm{keV}$ in the drift loss cone during the period from day 67 to day 76 of 1989 . STEEL (red circle) appears in the inner belt $(2<L<2.5)$ during the main phase of the day 68 storm whose $D_{s t}$ minimum reached $-101 \mathrm{nT}$. Note that STEEL is not clear at the day 72 great storm which reached the $D_{s t}$ minimum of $-589 \mathrm{nT}$, because the violent injection may prevent identifying STEEL. One of the possible mechanisms explaining STEEL would be radial transport processes from the slot region to the inner belt by both large convective electric fields and radial diffusion. The strong gradient of a phase space density at great storm may produce the fast radial transport. Thus, it may be expected that the strong convective electric field causes STEEL. Therefore, we first investigate the radial transport process under strong convective electric fields.

Figure $1 \mathrm{~b}$ shows the variation of convective electric fields at the magnetic equator during the same period with Fig. 1a. Using similar method of Mozer (1970), we converted the electric fields observed by the Akebono satellite to values of convective electric fields at the magnetic equator. Positive and negative electric fields mean dawn-dusk electric fields and dusk-dawn electric fields, respectively. The purple line in Fig. 1a indicates the inner edge of the outer belt. Here, the inner edge is defined as the location of more than 10000 $\left(\mathrm{cm}^{2} \mathrm{~s}\right.$ str) of electron flux at $300 \mathrm{keV}$. It can be seen that the location of inner edge of the outer belt concurrently varies with the strong convective electric fields, that is, the strong 


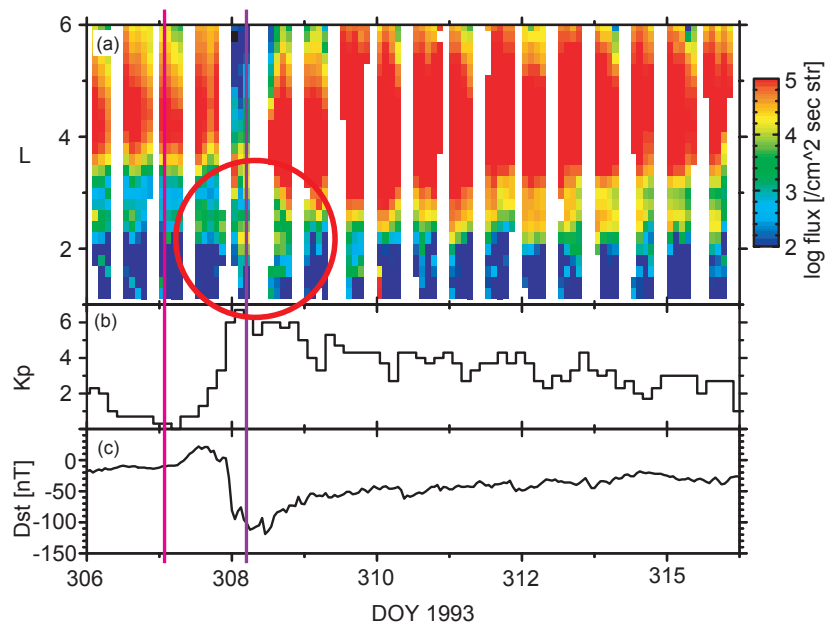

Fig. 2. An example of the electron flux enhancement in the inner belt during the period from 306 to 316 day of 1993 (a). $K_{p}$ (b) and $D_{s t}$ indices (c). The pink and purple lines correspond to the dynamic spectra in Figs. 3 and 4, respectively.

convective electric fields invade into the inner belt (around $L \sim 2$ ) with injection of outer belt electrons during the day 72 great storm, while the fields do not invade into the inner region at the day 68 storm. The strong convective electric fields more than $3 \mathrm{mV} / \mathrm{m}$ were not observed in the inner belt at the STEEL of the day 68 storm. Thus, we can conclude that the variations of convective electric fields do not always affect the STEEL.

\subsection{Plasma wave activity during STEEL}

Another possible mechanism for STEEL would be the stormtime precipitation of the trapped electrons from the inner belt. In this section, we examine the pitch angle scattering of the trapped electrons through wave-particle interactions.

A typical example of STEEL in the drift loss cone during the period from day 306 to day 316 of 1993 is shown in Fig. 2a. Figure 2b and c indicate the $K_{p}$ and $D_{s t}$ indices, respectively. The storm on day 307-308 was a storm which had the minimum $D_{s t}$ value of $-119 \mathrm{nT}$. In order to investigate wave-particle interactions during STEEL, we examined STEEL event and related plasma wave spectra. Dynamic spectra of plasma waves observed by the Akebono satellite at the dawn side are illustrated in Figs. 3 and 4. Figure 3 shows quiet time (before the storm) spectra for $20 \mathrm{~min}$ on day 307 (period shown by the pink bar in Fig. 2) during the $D_{s t}=-12 \mathrm{nT}$. Figure 4 shows the storm time spectra for $30 \mathrm{~min}$ on day 308 (period shown by the purple bar in Fig. 2) during the $D_{s t}=-96 \mathrm{nT}$. The horizontal axes in the two figures show the universal time (UT), magnetic local time (MLT), altitude (ALT), L-value ( $L$ ), and geomagnetic latitude (GLAT). The top panels of Figs. 3 and 4 represent the intensity of wave electric fields observed by the PWS in-

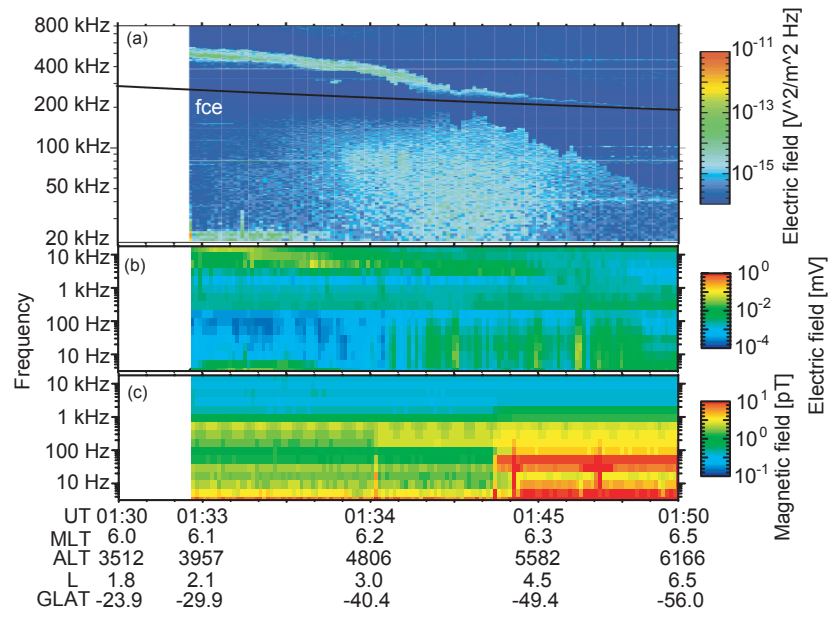

Fig. 3. Power spectral density of plasma waves observed by the Akebono satellite during a magnetic quiet time on day 307 of 1993. The solid line shows the local electron gyrofrequency. The top and middle panels exhibit the wave spectral electric field intensity by the PWS and the VLF instruments. The bottom panel indicates the wave spectral magnetic field intensity by the VLF/Akebono.

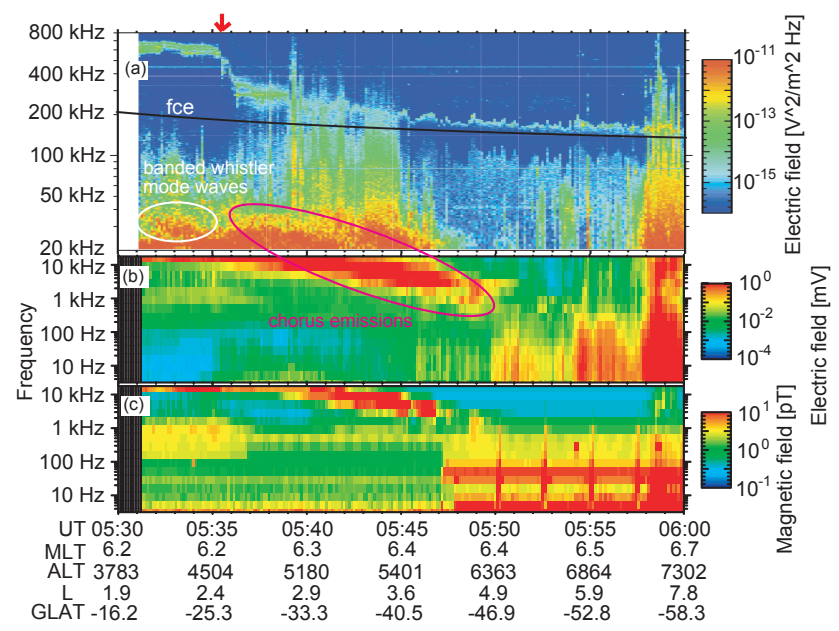

Fig. 4. Same as Fig. 3 but during the electron flux enhancement on day 308 of 1993. The red arrow shows the location of the plasmapause.

strument in the frequency range from 20 to $800 \mathrm{kHz}$, and the middle and bottom panels show the wave electric and magnetic field spectrograms in the frequency range from $3.16 \mathrm{~Hz}$ to $17.8 \mathrm{kHz}$ observed by the VLF instrument. The black line in the top panel shows the local electron cyclotron frequency $\left(f_{c e}\right)$. During the quiet time (Fig. 3), waves are rather inactive. The banded emissions at several hundred $\mathrm{kHz}$ as seen in the top panel of Fig. 3 are the upper hybrid resonance (UHR) emissions. Whistler mode waves are evident below $f_{c e}$ with considerably weak intensity as shown in Fig. 3. Strong wave activity is apparent during the storm time as shown in Fig. 4 . 


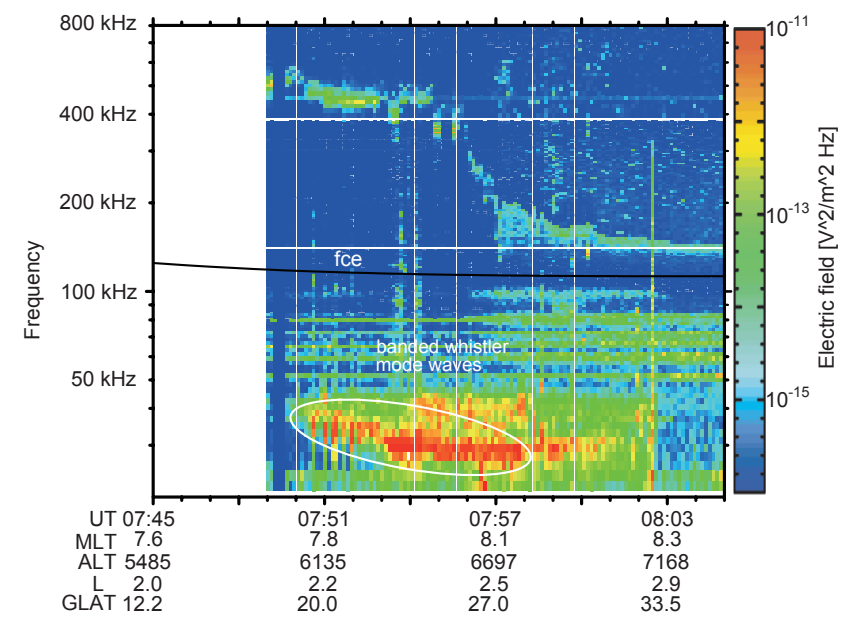

Fig. 5. Power spectral density of plasma waves observed by the Akebono satellite during day 101 of 1997 . The solid line shows the local electron gyrofrequency. The white circle indicates banded whistler mode waves.

The location of plasmapause in Fig. 4 can be determined to be $L=2.4$ (red arrow on the top of Fig. 4a), where the UHR frequency shows sudden decrease. Broad band electrostatic noise (BEN) is measured beyond $L \sim 6.6$. Strong chorus emissions and associated bursty electrostatic waves whose upper frequency exceed the UHR frequency are observed outside of the plasmapause ( $L=2.4-6.4$ ). These chorus emissions are considered to contribute to the acceleration of electron flux in the outer belt (e.g., Miyoshi et al., 2003; $\mathrm{Li}$ et al., 2007). Note that strong "banded-emissions" in the frequency range from 15 to $30 \mathrm{kHz}$ are detected in the inner belt region (pink circle in Fig. 4a and b) in the storm main phase. We notice that these emissions in the plasmasphere have not been reported except for Kobayashi (1997). The wave magnetic field intensities of these banded whistler mode waves are estimated to be over $10 \mathrm{pT}$. These banded whistler mode waves are typical in the plasmasphere and different from chorus emissions which appear outside the plasmapause (e.g., Horne et al., 2005; Miyoshi et al., 2007). Another typical example of the banded whistler mode waves at the day 101 of 1997 is shown in Fig. 5. The minimum $D_{s t}$ value on that day was $-82 \mathrm{nT}$. The characteristics of the banded whistler mode waves are band-type spectra and shows sometimes frequency drift. Therefore, it is apparent that these banded whistler mode waves are natural generated whistler mode waves that can be distinguished from VLF transmitters. Plasmaspheric hiss emissions, which are broadband whistler mode emissions with peak spectral intensity at a few hundred Hz (e.g., Thorne et al., 1973; Meredith et al., 2004; Bortnik et al., 2008), are commonly detected in the inner belt as seen in Fig. 4c (05:30-05:36UT). The frequency of these plasmaspheric hiss emissions is quite different from that of the banded whistler emissions.

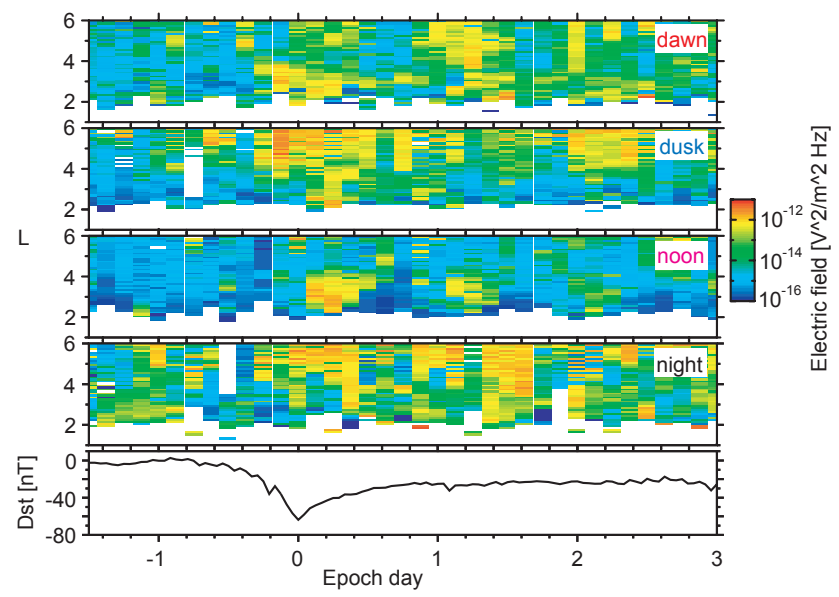

Fig. 6. Local time dependence of the superposed wave electric field amplitude in the frequency range from 25 to $33 \mathrm{kHz}$ during the period from 1992 to 1994 . The selected storms are 37 small and moderate ones. The zero epoch is taken to be the minimum $D_{s t}$.

\subsection{Statistical analysis of banded whistler mode waves}

In order to clarify storm-time characteristics of whistler mode emissions, Fig. 6 shows a superposed epoch analysis for 37 storms during the period when the wave data in the inner belt were available from 1992 to 1994 . The selected storms are small and moderate storms $\left(D_{s t}=-30 \mathrm{nT}\right.$ $\sim-100 \mathrm{nT})$ : small and moderate storms are defined as minimum $D_{s t}$ of -30 to $-100 \mathrm{nT}$ in Tadokoro et al. (2007). The zero epoch is taken to be the minimum $D_{s t}$. The analyzed frequency range of whistler mode emissions is $25-33 \mathrm{kHz}$. It should be noted that, in this analysis, the waves in $25-33 \mathrm{kHz}$ include not only the banded whistler mode waves of interest but also VLF transmitters, various electrostatic waves, and BEN at the higher L-region. The superposed epoch analysis shows that the wave intensity at the dawn side increases in the low L-region $(<4)$ during the main phase, and the enhanced wave activity lasts for about 1 day. In the recovery phase, the wave activity in the lower L-region increases at the dusk and noon sides. The waves observed in the low Lregion probably include the banded whistler mode waves as shown in Figs. 5 and 6. Comparing to the characteristics of STEEL by Tadokoro et al. (2007, Fig. 4), the start time and duration of STEEL almost consistent with those of the wave intensity increase.

The whistler mode wave activity in the inner and the outer belt at a few tens of $\mathrm{kHz}$ was first reported by Kobayashi (1997). The report showed that intensities of such whistler mode waves observed by the PWS/Akebono increase during a storm, and clarified the characteristics of the latitudinal distribution of the whistler mode waves as shown in Fig. 7 (private communication with Kobayashi). Figure 7 indicates a meridian distribution of the waves in the frequency range from 24 to $34 \mathrm{kHz}$ during the period from 1989 to 1995 . 


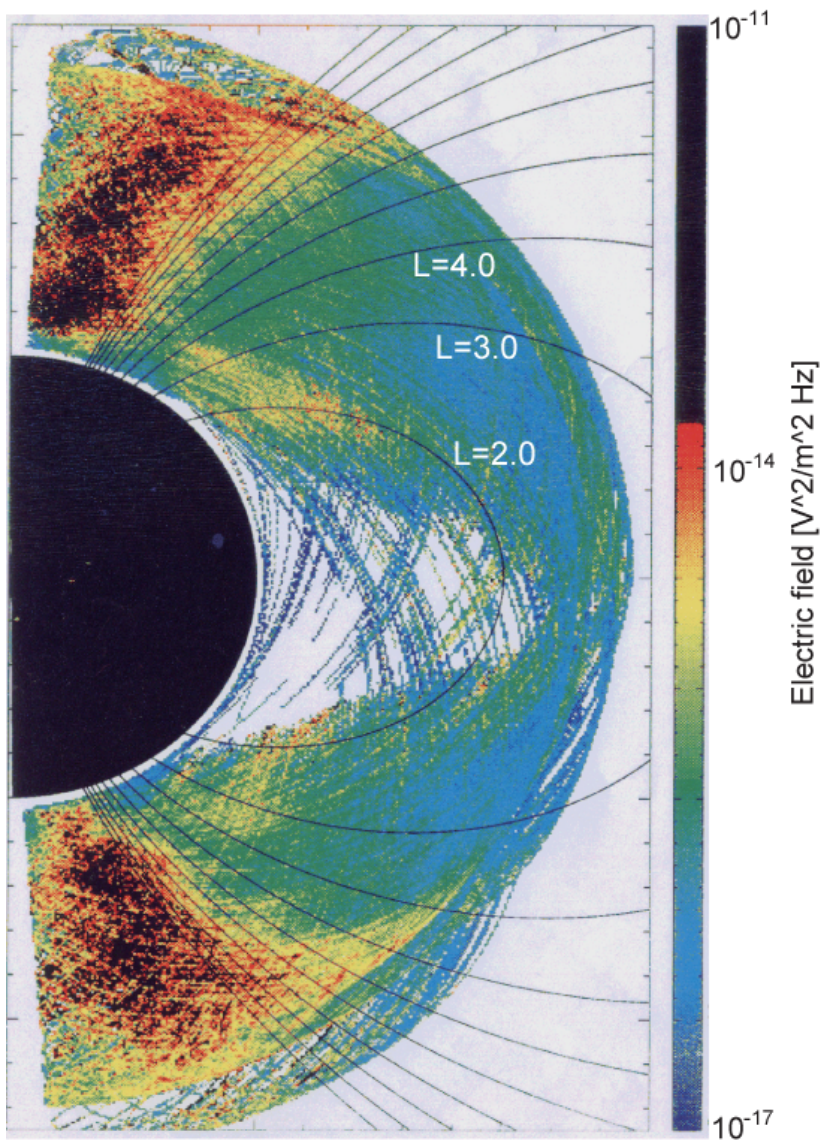

Fig. 7. Latitudinal distribution of the averaged electric field spectral intensity in the frequency range from 24 to $34 \mathrm{kHz}$ during the period from 1989 to 1995 (courtesy of Kobayashi, 1997).

BENs are detected in the higher magnetic latitudes. Note that the enhanced wave activity is detected in the mid-latitudes from $L \sim 2$ to $\sim 3$, which are the banded whistler mode waves.

From the above investigation, it is expected that STEEL is possibly related with the enhancement of the banded whistler mode waves, and caused by pitch angle scattering through wave-particle interactions. In the next section, we investigate this possibility of wave-particle interactions by the numerical calculations.

\subsection{Numerical calculation}

In order to investigate the possibility that the observed STEEL is caused by the pitch angle scattering, we examine the time evolution of pitch angle distribution of inner belt electrons during a storm. Pitch angle diffusion coefficients for Landau $(n=0)$ and cyclotron resonances $(n= \pm 5)$ are calculated following a previous model by Albert (1999). The bounce-averaged relativistic quasi-linear diffusion equation is given (e.g., Lyons et al., 1972), as

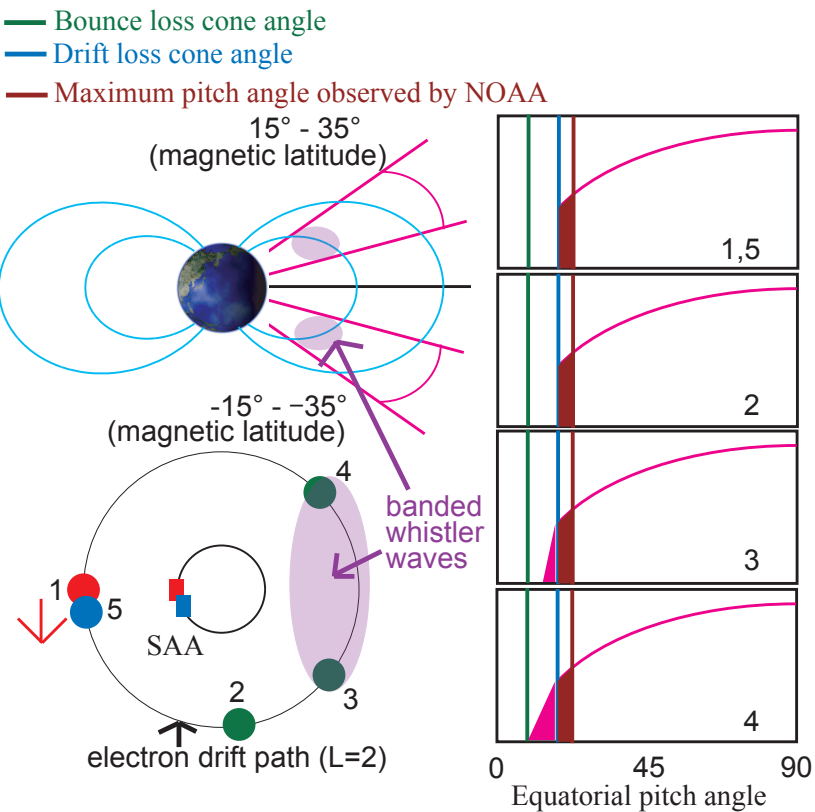

Fig. 8. Schematic illustration of method of numerical calculation. The middle and bottom panels on the left indicate the region of the banded whistler mode waves and an electron trajectory along the one drift path at $L=2.0$, respectively. The right panels show schematic pitch angle distributions corresponding to the numbers in the bottom panel on the left. The solid green, blue, and brown lines exhibit the bounce loss cone angle, drift loss cone angle, and the maximum pitch angle observed by the NOAA 12 satellite, respectively.

$$
\frac{\partial f}{\partial t}=\frac{1}{p^{2} T\left(\alpha_{e q}\right) \sin 2 \alpha_{e q}} \frac{\partial}{\partial \alpha_{e q}} D_{\alpha \alpha} T\left(\alpha_{e q}\right) \sin 2 \alpha_{e q} \frac{\partial f}{\partial \alpha_{e q}}
$$

where $f$ is the zero-order spatially uniform particle distribution, $p$ is the momentum, $D_{\alpha \alpha}$ is the bounce averaged pitch angle diffusion coefficient, $\alpha_{e q}$ is the equatorial pitch angle, and $T(\alpha) \approx 1.30-0.56 \sin \alpha_{e q}$ is given by Hamlin et al. (1961).

Figure 8 illustrates the calculation procedure. The left panels show the schematic meridional distribution of the banded whistler mode waves and an electron drift trajectory during a drift period. We calculate Eq. (1) during one drift period by using the wave characteristics observed during the storm main phase. The drift period of a $300 \mathrm{keV}$ electron along $L=2.0$ is estimated to be about $1 \mathrm{~h}$ under the consideration for a dipole magnetic field and co-rotational electric field. The statistical result in Fig. 6 shows that the banded whistler mode waves appear only at the dawn sector (from 03:00 to 09:00 LT) during the main phase. This means that drifting electrons experience pitch angle scattering over a quarter of one drift path. Following the observation as shown in Fig. 7, we assume that the banded whistler mode waves distribute from $15^{\circ}$ to $35^{\circ}$ magnetic latitudes in both hemispheres. The schematic panels in the right of Fig. 8 show pitch angle distributions at the five locations indicated in the bottom left 


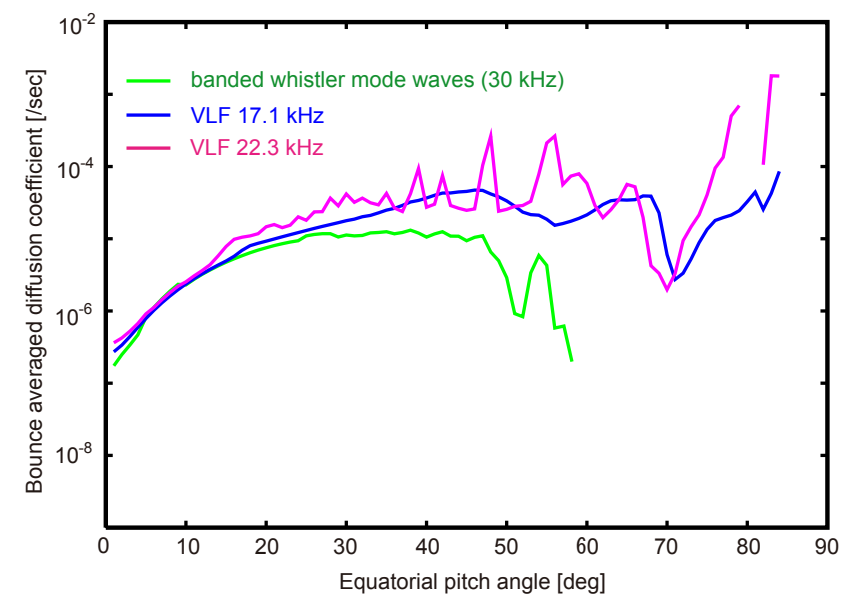

Fig. 9. Bounce averaged pitch angle diffusion coefficients for various whistler mode waves with $300 \mathrm{keV}$ electron at $L=2.0$, NOAA altitude. The solid green, blue, and pink lines indicate, band whistler mode waves, VLF transmitters at $17.1 \mathrm{kHz}$, and $22.3 \mathrm{kHz}$, respectively.

panel. The numbers in the right panels correspond to those of the bottom left panel. The solid green, blue, and brown lines in the right panels indicate the bounce loss cone angle, drift loss cone angle $\left(22.5^{\circ}\right)$, and maximum equatorial pitch angle $\left(25.1^{\circ}\right)$ observed by the NOAA 12 altitude, respectively. We used a constant bounce loss cone angle of $17^{\circ}$ although the angle depends on longitudes, and we derived the drift loss cone, bounce loss cone, and maximum equatorial pitch angles from magnetic fields and pitch angles at the magnetic equator and mirror altitude. The brown color region in the right panels of Fig. 8 shows pitch angle region with stably trapped electrons which are observed only at the South Atlantic Anomaly (SAA) region by NOAA. The calculation starts from the meridian of just east of the SAA (point 1 ) and ends at the point 5 via point 2,3, and 4 along the electron drift trajectory. At the point 1 electrons in the drift loss cone precipitate into the atmosphere as illustrated in the right panel of Fig. 8 when they pass over in the SAA region. The electrons around the point 2 do not suffer any pitch angle scattering because of no banded whistler mode waves there. The electrons at the point 3 encounter the banded whistler mode waves and begin to suffer the pitch angle scattering. Some electrons diffuse from the trapping distribution (brown color region in the right panel in Fig. 8) to the drift loss cone distribution (pink color region in the right panel in Fig. 8) as the result of the pitch angle scattering. As long as the electrons pass through the region of the banded whistler mode waves, the electrons continue to suffer pitch angle scattering and diffuse into the drift loss cone. The electrons in the drift loss cone accumulate as the result of such pitch angle scattering, and precipitate into the atmosphere again when they return to the SAA at the point 5 . Thus, it is expected that the amount of electrons in the drift loss cone that are precip-

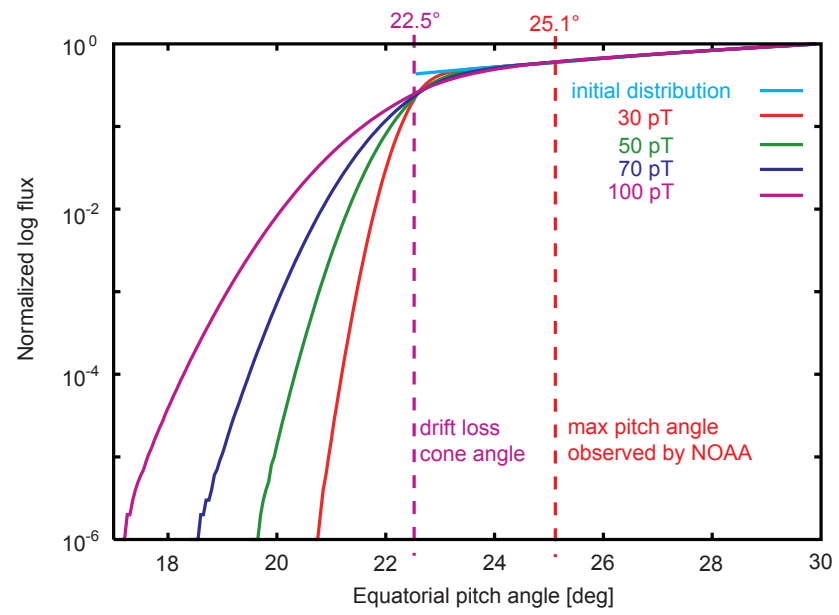

Fig. 10. Pitch angle distributions after one drift period for various wave magnetic field amplitudes, $30,50,70$, and $100 \mathrm{pT}$ at $L=2.0$. The initial distribution is adopted from the electron flux at $341 \mathrm{keV}$ observed by the CRRES satellite. The dashed red and purple lines indicate the maximum equatorial pitch angle observed by NOAA and the drift loss cone angle, respectively.

itating into the atmosphere would correspond to the STEEL observed by NOAA.

Figure 9 indicates the bounce averaged pitch angle diffusion coefficients of $300 \mathrm{keV}$ electrons for various whistler mode waves at $L=2.0$, NOAA altitude. The green, blue, and pink lines correspond to the calculated coefficients for the banded whistler mode waves of interest at $30 \mathrm{kHz}$ in this study, and VLF transmitters at $17.1 \mathrm{kHz}$ and $22.3 \mathrm{kHz}$, respectively. Wave propagation angle, width of propagation angle, and wave amplitude for each wave are taken to be $45^{\circ}$, $22.5^{\circ}$, and $10 \mathrm{pT}$, respectively. These parameters of plasmaspheric hiss, VLF transmitters are based on those of Meredith et al. (2007) and Abel and Thorne (1998a, b, 1999), respectively. The bandwidth of the banded whistler mode waves is set to be $30 \mathrm{kHz}$ based on the observational data. Plasmaspheric hiss emissions in Fig. 9 are not shown because they do not have pitch angle diffusion coefficients for $300 \mathrm{keV}$ electrons at $L=2$ as suggested by Meredith et al. (2007). On the other hand, the banded whistler mode waves and VLF transmitters show the significant diffusion coefficients around the loss cone. Although whistler mode waves from the VLF transmitters can resonate with electrons, they are not likely to cause the time variation of STEEL because intensities of VLF transmitters are stable and does not depend on storm activity. Therefore, STEEL can be concluded to be caused by pitch angle scattering due to the banded whistler mode waves.

Lightning-generated whistler mode waves are also filled in the plasmasphere and have an important role in the loss processes of inner belt electrons (Blake et al., 2001; Rodger et al., 2003, 2004, 2007). However, it is unlikely that theses 
whistler mode waves always appear in the inner radiation belt during a storm as discusses in this study. Therefore, we do not discuss lightning-generated whistler mode waves any more.

We examine the observation by comparing the ratio of the amount of electron flux at $300 \mathrm{keV}, L=2$ in the drift loss cone to that in the trapping region after one drift period $(1 \mathrm{~h})$. The ratio obtained from observation was 1:10 (not shown). Figure 10 exhibits calculated pitch angle distributions during one drift period for the cases of rather strong wave amplitudes of 30,50,70, and $100 \mathrm{pT}$ since the observed wave amplitudes were much greater than $10 \mathrm{pT}$. The horizontal axis indicates the equatorial pitch angle from $17^{\circ}$ to $30^{\circ}$. The initial distribution is adopted to that of the $341 \mathrm{keV}$ electron flux observed by the CRRES satellite at the quiet time (light blue line). Note that the electron flux is normalized at an equatorial pitch angle of $30^{\circ}$. We assume that electrons at $17^{\circ}$ and $22.5^{\circ}$ (equatorial pitch anlge) precipitate into the atmosphere during one bounce period, and one drift period, respectively, that is, electrons in the drift loss cone have loss rates due to interaction with the atmosphere. These loss rates in the drift loss cone are interpolated from the loss rates of $17^{\circ}$ and $22.5^{\circ}$. The dashed red and purple lines show the maximum equatorial pitch angle observed by NOAA and the drift loss cone angle, respectively. The electron flux below the drift loss cone angle is set to be zero at the calculation start time (point 1 in Fig. 8) because of loss into the atmosphere above the SAA. The solid lines indicate the calculated pitch angle distributions after one drift period for various wave amplitudes. The electron flux with the pitch angle greater than the drift loss cone angle is almost constant after one drift period. This suggests that the loss of the trapped electrons is small. On the other hand, electron flux in the drift loss cone after one drift period increases significantly with increasing of wave amplitudes. The calculated ratios of electron flux in the drift loss cone to that in the trapping region are 1:21.2, 1:12.0, 1:8.0, and 1:5.2 for the wave amplitudes of 30, 50, 70, and $100 \mathrm{pT}$. These wave amplitudes have same order as the observed wave amplitudes (over $10 \mathrm{pT}$ as referred to above). With regard to the calculated ratios, 50$70 \mathrm{pT}$ are roughly consistent with the observational result (1:10 as referred to above). Estimated lifetime of electrons at $300 \mathrm{keV}, L=2$ using the estimated coefficients with a wave amplitude of $100 \mathrm{pT}$ are approximately 200 days. This lifetime is almost comparable with combination among whistler mode hiss, lightning whistler and VLF transmitter (Fig. 10 of Abel and Thorne, 1998a), so that it is expected that the banded-whistler mode waves have large impact of distribution of radiation belt electrons.

\section{Summary}

We have examined Storm Time inner belt Electron Enhancement at the Low altitude (STEEL) reported by Tadokoro et al. (2007). Radial transport process by convective electric fields was excluded as the mechanism of the STEEL since strong fields are not observed at the region of STEEL during storms. In order to examine the possibility of pitch angle scattering due to wave-particle interactions, we have clarified the observed relation between the electron flux enhancement and the plasma wave intensity. Intense plasmaspheric hiss and the banded whistler mode waves $(\sim 30 \mathrm{kHz})$ are observed by the PWS/Akebono in the inner belt during STEEL. The calculated diffusion coefficients indicated that the resonance is possible with the banded whistler mode waves. The banded whistler mode waves appear with strong intensity in the main phase at the dawn side, and last for about 1 day. This start time and duration of the banded whistler mode waves are almost consistent with those of STEEL. Based on these evidences, we concluded that STEEL is caused by the pitch angle scattering through wave-particle interactions with the storm time banded whistler mode waves. Furthermore, we have calculated the time evolution of pitch angle distribution through wave-particle interactions with the banded whistler mode waves with amplitude of several tens of $\mathrm{pT}$ over one drift period. The estimated result of the ratio of electron flux in the drift loss cone to that in the trapping distribution is roughly consistent with the observed results.

Acknowledgements. We thank K. T. Asai for useful discussions regarding problems related to contamination of the MEPED instrument. The $D_{s t}$ and $K_{p}$ indices are provided from the World Data Center for Geomagnetism (WDC-C2) in Kyoto University. NOAA satellite data are provided through the WDC-C2 for Aurora, National Institute of Polar Research, Japan. CRRES satellite data are provided through the Coordinated Data Analysis Web (CDAWeb/NASA).

Topical Editor I. A. Daglis thanks two anonymous referees for their help in evaluating this paper.

\section{References}

Abel, B. and Thorne, R. M.: Electron scattering loss in Earth's inner magnetosphere, 1, Dominant physical processes, J. Geophys. Res., 103, 2385-2396, 1998a.

Abel, B. and Thorne, R. M.: Electron scattering loss in Earth's inner magnetosphere, 2, Sensitivity to model parameters, J. Geophys. Res., 103, 2397-2407, 1998b.

Abel, B. and Thorne, R. M.: Correction to "Electron scattering loss in Earth's inner magnetosphere, 1, Dominant physical processes" and "Electron scattering loss in Earth's inner magnetosphere, 2, Sensitivity to model parameters", J. Geophys. Res., 104, 46274628, 1999.

Albert, J. M.: Analysis of quasi-linear diffusion coefficients, J. Geophys. Res., 104, 2429-2441, 1999.

Baker, D. N., Kanekal, S. G., Li, X., Monk, S. P., Goldstein, J., and Burch, J. L.: An extreme distortion of the Van Allen belt arising from the Hallowe'en solar storm in 2003, Nature, 432, 878-881, 2004.

Blake, J. B., Inan, U. S., Walt, M., Bell, T. F., Bortnik, J., Chenette, D. L., and Christian, H. J.: Lightning-induced energetic electron 
flux enhancements in the drift loss cone, J. Geophys. Res., 106, 29733-29744, 2001.

Bortnik, J., Thorne, R. M., and Meredith, N. P.: The unexpected origin of plasmaspheric hiss from discrete chorus emissions, Nature, 452, 62-66, doi:10.1038/nature06741, 2008.

Bostrom, C. O., Beall, D. S., and Armstrong, J. C.: Time History of the Inner Radiation Zone, October 1963 to December 1968, J. Geophys. Res., 75, 1246-1256, 1970.

Brautigam, D. H. and Albert, J. M.: Radial diffusion analysis of outer radiation belt electrons during the October 9 1990, magnetic storm, J. Geophys. Res., 105, 291-309, 2000.

Datlowe, D. W.: Differences between transmitter precipitation peaks and storm injection peaks in low-altitude energetic electron spectra, J. Geophys. Res., 111, A12202, doi:10.1029/2006JA011957, 2006.

Friedel, R. H. W., Reeves, G. D., and Obara, T.: Relativistic electron dynamics in the inner magnetosphere - a review, J. Atmos. Sol. Terr. Phys., 64, 265-282, 2002.

Gusev, A. A., Kohno, T., Martin, I. M., Pugacheva, G. I., Turtelli Jr., A., Tylka, A. J., and Kudela, K.: Injection and fast radial diffusion of energetic electrons into the inner magnetosphere, Planet. Space Sci., 43, 1131-1134, 1995.

Hamlin, D. A., Karpulus, R., Vik, R. C., and Watson, K. M.: Mirror and azimuthal drift frequencies for geomagnetically trapped particles, J. Geophys. Res., 66, 1-4, 1961.

Hayakawa, H., Okada, T., Ejiri, M., Kadokura, A., Kohno, Y.-I., Maezawa, K., Machida, S., Matsuoka, A., Mukai, T., Nakamura, M., Nishida, A., Obara, T., Tanaka, Y., Mozer, F. S., Haerendel, G., and Tsuruda, K.: Electric Field Measurement on the Akebono (EXOS-D) Satellite, J. Geomagn. Geoelectr., 42, 371-384, 1990.

Horne, B. R., Thorne, R. M., Glauert, S. A., Albert, J. M., Meredith, N. P., and Anderson, R. R.: Timescale for radiation belt electron acceleration by whistler mode chorus waves, J. Geophys. Res., 110, A03225, doi:10.1029/2004JA010811, 2005.

Imhof, W. L., Gaines, E. E., and Reagan, J. B.: Evidence for the Resonance Precipitation of Energetic Electrons From the Slot Region of the Radiation Belts, J. Geophys. Res., 79, 3141-3146, 1974.

Inan, U. S., Bell, T. F., and Chang, H. C.: Particle precipitation induced by short-duration VLF waves in the magnetosphere, J. Geophys. Res., 87, 6243-6246, 1982.

Kikuchi, T. and Evans, D. S.: Energetic electrons observed by NOAA-6 over Japan $(\mathrm{L}=1.3)$ at the time of geomagnetic storm on February 8-9, 1986, Proc. Res. Inst. Atmos., Nagoya Univ., 36, 137-149, 1989.

Kimura, I., Hashimoto, K., Nagano, I., Okada, T., Yamamoto, M., Yoshino, T., Matsumoto, H., Ejiri, M., and Hayashi, K.: VLF observations by the Akebono (EXOS-D) satellite, J. Geomagn. Geoelectr., 42, 459-478, 1990.

Kobayashi, H.: Wave-particle interactions between LF waves and energetic particles in the outer belt (in Japanese), Master thesis, Tohoku Univ., Sendai, 1997.

Li, W., Shprits, Y. Y., and Thorne, R. M.: Dynamic evolution of energetic outer zone electrons due to wave-particle interactions during storms, J. Geophys. Res., 112, A10220, doi:10.1029/2007JA012368, 2007.

Lyons, L. R., Thorne, R. M., and Kennel, C. F.: Pitch angle diffusion of radiation belt electrons within the plasmasphere, J. Geophys. Res., 77, 3455-3474, 1972.
Meredith, N. P., Horne, R. B., Thorne, R. M., Summers, D., and Anderson, R. R.: Substorm dependence of plasmaspheric hiss, J. Geophys. Res., 109, A06209, doi:10.1029/2004JA010387, 2004.

Miyoshi, Y., Morioka, A., Obara, T., and Nagai, T.: Dynamics of the energetic particles during the storm main phase - EXOS-D (Akebono) and NOAA observations, Eos. Trans. AGU, 83(22), West. Pac. Geophys. Meet. Suppl., Abstract SP21A-01, 2002.

Miyoshi, Y., Morioka, A., Obara, T., Misawa, H., Nagai, T., and Kasahara, Y.: Rebuilding process of the outer radiation belt during the 3 November 1993 magnetic storm: NOAA and Exos-D observations, J. Geophys. Res., 108, 1004, doi:10.1029/2001JA007542, 2003.

Miyoshi, Y., Jordanova, V., Morioka, A., and Evans, D.: Solae cycle variation of the electron radiation belts: Observations and Radial diffusion simulation, Space Weather, 2, S10S02, doi:10.1029/2004SW000070, 2004.

Miyoshi, Y. and Kataoka, R.: Ring current ions and radiation belt electrons during geomagnetic storms driven by coronal mass ejections and corotating interaction regions, Geophys. Res. Lett., 32, L21105, doi:1029/2005GL024590, 2005.

Miyoshi, Y., Morioka, A., Kataoka, R., Kasahara, Y., and Mukai, T.: Evolution of the outer radiation belt during the November 1993 storms driven by corotating interaction regions, J. Geophys. Res., 112, A05210, doi:10.1029/2006JA012148, 2007.

Morioka, A., Miyoshi, Y., and Obara, T.: Dynamics of Energetic Particles in the Inner Radiation Belt During Magnetic Storms, International Space Environment Conference 2001, Queenstown, New Zealand, 2001.

Mozer, F. S.: Electric field mapping in the ionosphere at the equatorial plane, Planet. Space Sci., 18, 259-263, 1970.

Oya, H., Morioka, A., Kobayashi, K., Iizima, M., Ono, T., Miyaoka, H., Okada, T., and Obara, T.: Plasma wave observation and stimulation experiments (PWS) using Akebono (EXOS-D) satellite Instrumentation and initial results including discovery of the high altitude equatorial plasma turbulence, J. Geomagn. Geoelectr., 42, 411-442, 1990.

Parrot, M., Santolík, O., Cornilleau-Wehrlin, N., Maksimovic, M., and Harvey, C.: Magnetospherically reflected chorus waves revealed by ray tracing with CLUSTER data, Ann. Geophys., 21, 1111-1120, 2003, http://www.ann-geophys.net/21/1111/2003/.

Raben, V. J., Evans, D. S., Sauer, H. H., Sahm, S. R., and Huynh, M.: TIROS/NOAA satellite space environment monitor data archive documentation: 1995 update, NOAA Tech. Memo. ERL SEL-86, 1995.

Rodger, C. J., Clilverd, M. A., and McCormick, R. J.: Significance of lightning-generated whistlers to inner radiation belt electron lifetimes, J. Geophys. Res., 108, 1462, doi:10.1029/2003JA009906, 2003.

Rodger, C. J., McCormick, R. J., and Clilverd, M. A.: Testing the important of precipitation loss mechanisms in the inner radiation belt, Geophys. Res. Lett., 31, L10803, doi:1029/2004GL019501, 2004.

Rodger, C. J., Enell, C.-F., Turunen, E., Clilverd, M. A., Thomson, N. R., and Verronen, P. T.: Lightning-driven inner radiation belt energy deposition into the atmosphere: implications for ionisation-levels and neutral chemistry, Ann. Geophys., 25, 1745-1757, 2007, http://www.ann-geophys.net/25/1745/2007/. 
Shprits, Y. Y., Elkington, S. R., Meredith, N. P., and Subbotin, D. A.: Review of modeling of losses and sources of relativistic electrons in the outer radiation belt I: Radial transport, J. Atmos. Sol. Terr. Phys., 70, 1679-1693, doi:10.1016/j.jastp.2008.06.008, 2008a.

Shprits, Y. Y., Subbotin, D. A., Meredith, N. P., and Elkington, S. R.: Review of modeling of losses and sources of relativistic electrons in the outer radiation belt II: Local acceleration and loss, J. Atmos. Sol. Terr. Phys., 70, 1694-1713, doi:10.1016/j.jastp.2008.06.014, 2008b.
Tadokoro, H., Tsuchiya, F., Miyoshi, Y., Misawa, H., Morioka, A., and Evans, D. S.: Electron flux enhancement in the inner radiation belt during moderate magnetic storms, Ann. Geophys., 25, 1359-1364, 2007, http://www.ann-geophys.net/25/1359/2007/.

Thorne, R. M., Smith, E. J., Burton, R. K., and Holzer, R. E.: Plasmaspheric hiss, J. Geophys. Res., 78, 1581-1596, 1973.

Walt, M. and Newkirk, L. L.: Addition to Investigation of the Decay of the Starfish Radiation Belt, J. Geophys. Res., 71, 3265-3266, 1966. 